\title{
Reflexiones Oriqinales
}

\section{Etnoeducación}

Lic. GIGSON UseChe GonzÁLEZ*

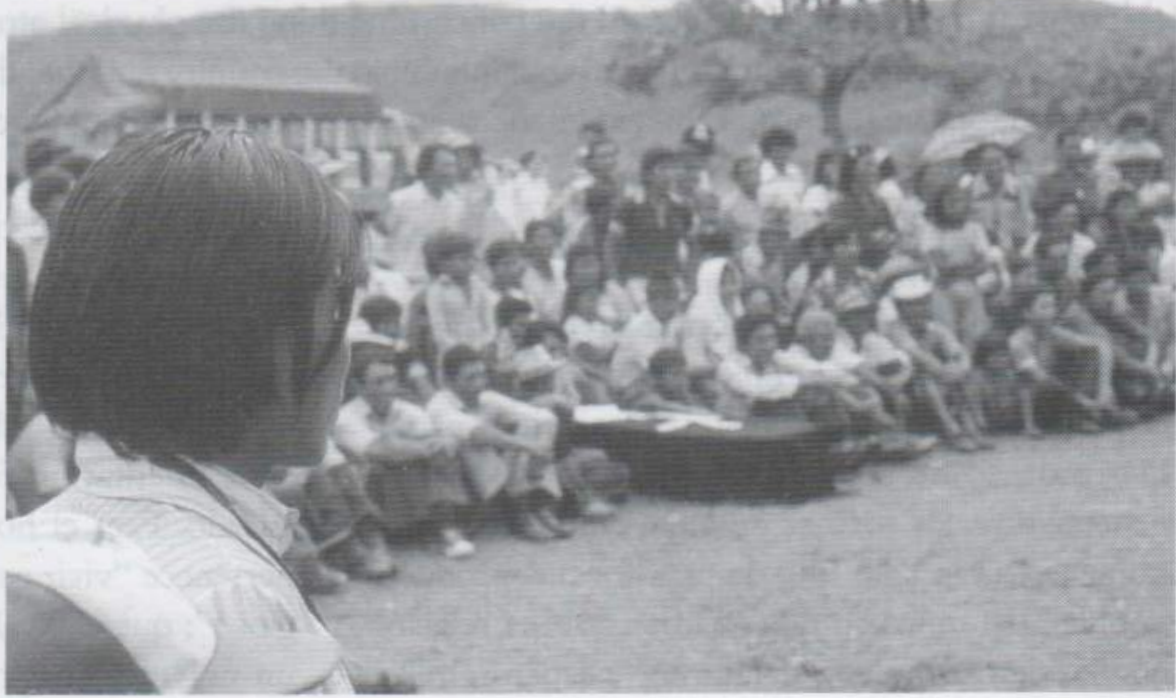

Por: Mario G. Loaiza Padilla

\section{Resumen}

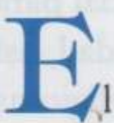

presente artículo forma parte de una serie de escritos y reflexiones que se vienen elaborando dentro del proyecto Acompañamiento y Asesoría a las Comunidades Indígenas y Afroclombianas en los Procesos de Etnoeducación y Educación Propia, implementado en la Práctica Pedagógica del Departamento de Educación Física. Inicialmente se describe una introducción que hace referencia a la cosmogonía de las comunidades indígenas y afrocolombianas; seguidamente se expone a manera de justificación, una interpretación de los modelos educativos hegemónicos, asimilacionistas, excluyentes y discriminatorios que se han aplicado en el país, sin tener en cuenta la multietnicidad, la pluriculturalidad de los colombianos $y$, finalmente, se indica lo concerniente a la etnoeducación física como una propuesta que incluya a los diferentes grupos étnicos que habitan en el país y que poseen diversas concepciones, simbolizaciones, significados de las experiencias y prácticas corporales que conforman sus planes de vida.

Fecha de recepción: agosto 5 de 2003 - Fecha de aceptación: septiembre 17 de 2003

Palabras clave: etnoeducación, etnoeducación física, educación propia, planes de vida, derecho social, hecho social, educación hegemónica, modelo asimilacionista.

\section{Abstract}

The present article forms part of a series of writings and reflections that are come elaborating inside the project Accompaniment and consultantship to the indigenous communities and afroclombianas in the 
processes of etnoeducación and own education, implemented to the interior of the pedagogic practice of the Department of Physical Education. Initially an introduction is described that makes reference to the cosmogony of the indigenous communities and afrocolombianas; subsequently it is exposed by way of justification, an interpretation of the educational models hegemonic, asimilate, excluding and discriminatory that have been applied in the country, without keeping in mind the multiethnic, the pluriculture of the colombians and, finally, is indicated the concerning thing the physical etnoeducation as a proposal that it includes to the different ethnic groups that inhabit in the country and that they possess diverse conceptions, symbolizations, meanings of the experiences and corporal practices that conform its plans of life.

\section{Introducción}

Si se parte de la concepción de la vida como una manifestación de las complejas contradicciones de las fuerzas del universo en su permanente y evolutivo proceso de construcción y destrucción para generar diversas formas de vida, entonces la vida humana es tan solo una de esas formas producto también del universo que guarda estrecha relación con las demás que se originaron y existieron millones de años antes de que apareciera la humana.

Sin embargo, la herencia de la conquista y colonización europea nos cambia de tajo la concepción e interpretación del universo, y del ser humano como parte de él, y nos impone la filosofía occidental, que ha marcado derroteros de comprensión, generalmente enmarcados dentro de las ciencias positivistas; no obstante, los diferentes pueblos y culturas, desde la prehistoria y a través del tiempo, han generado formas propias de comprender la dinámica universo - humanidad, especialmente en dos dimensiones fundamentales para la existencia: el tiempo y el espacio.

El significado del tiempo, para las culturas andinas, africanas y todas aquellas que guardan su memoria, el significado del tiempo estuvo siempre íntimamente ligado con los ciclos del universo, de la vida, del ser, de tal manera que el tiempo no fue concebido como una linealidad de futuro, presente y pasado sino, por el contrario, como una curva cíclica.

Según María Cristina Navarrete, citando a Jonh Mbiti (1995), la idea africana del tiempo se constituye en una de las claves para alcanzar la comprensión de los conceptos básicos religiosos y filosófi- cos. Para los africanos el fenómeno religioso es concomitante a la existencia del ser humano, el cual alcanza la plenitud en la ancianidad; cuando está mas cerca de sus antepasados y se encuentra, por lo tanto, más integrado a la vida espiritual. Esto explica la orientación fundamental del tiempo hacia el pasado. Para el hombre y la comunidad a la cual pertenece "vivir es estar inmerso en un drama religioso"; que equivale a decir que el hombre vive en un universo religioso, del cual sus descendientes, de manera similar, encuentran en la muerte la continuidad de la vida. El lugar físico en que se entierran los muertos da lugar al espacio en el que construyen su territorialidad, como factor esencial para consolidación de las familias de la comunidad, así como de todas las estructuras sociales y religiosas que viabilizan sus proyectos y planes de vida.

El tiempo, desde ésta cosmovisión, más que una cifra numérica, medida y registrada en una máquina como se hace en la modernidad, es la sucesión de acontecimientos en la vida individual y comunitaria, privilegiando una visión que se orienta más hacia el pasado que bacia el futuro. Los hechos sociales producidos atrás y quienes los protagonizaron son rememorados a través de todas las expresiones trasmitidas por medio de la palabra, la música y el cuerpo (expresiones que aunque tienen una base fisiológica son más expresiones de la espiritualidad en su concepción cosmogónica), dejando poco lugar a la preocupación por lo que aún no ha sucedido y por lo tanto no es real.

El tiempo presente o real se basa entonces, en las vivencias y experiencias concretas inscritas en patrones vitales y culturales como los nacimientos y fallecimientos, los pasos hacia la juventud, la 
adultez, la vejez, los ciclos climáticos y productivos en concordancia con los de la naturaleza y los seres vivos (los ciclos lunares, el devenir del mar, las cosechas y las subiendas, etc.); inmersos dentro de los ritos y fiestas, que marcan el momento en el cual el ser espiritual celebra el ritmo del orden natural, interrumpiendo la cotidianidad y dando paso a la continuidad.

Con relación específica a la concepción del espacio, afirma John Mbiti (1995), en el caso de los africanos, "lo que les interesa es el espacio geográfico de sus vivencias, expresión concreta del presente y del pasado. Por esta razón los africanos están específicamente vinculados a la tierra que les proporciona lo necesario para la subsistencia y a la vez los vincula místicamente con los difuntos".

En el caso de las comunidades afrodescendientes ancestrales y actuales, la concepción del espacio se expresa materialmente en la construcción de territorialidades. Para las primeras, en los nichos naturales periféricos en los que se pudo consolidar la identidad cultural, mantener sus tradiciones y su relación con los muertos. Para las segundas, que habitan en los nuevos paisajes, urbanos generalmente colonizados por intención ajena, como en el caso de los desplazamientos forzados o por voluntad propia, la territorialidad ya no se construye sobre el territorio, porque no lo hay, pero si sobre la tradición, la oralidad y el pasado.

En ese tránsito espacial, muchas veces se rezaga la identidad cultural, al ser avasallada por el ímpetu de la ciudad, a pesar de ello, y por ley natural del cosmos, siempre se regresa a la tierra natal, a la ancestralidad, porque siempre permanece en la afrodescendencia el trabajo permanente de la memoria en donde conserva viva la cultura y adquiere diferentes expresiones a través del mito y la leyenda, para ser narradas, cantadas o llevadas a diferentes lenguajes y formas materiales.

Con respecto a las comunidades indígenas ancestrales, resguardadas desde la colonia y que conservan aún sus territorios, luchan contra el des- pojo que permanentemente hacen de él los terratenientes, narcotraficantes, empresarios agroindustriales y colonos campesinos llamados por el taita Lorenzo Muelas: "los hijos del viento": resisten toda clase de embates y permanecen ahí, donde sus ancestros le legaron la tierra, la tradición y la cultura, recreando o conservando el proyecto de vida heredado del pensamiento del mundo andino y que la llamada racionalidad de Occidente no ha podido respetar y mucho menos entender.

$\mathrm{Al}$ igual que los integrantes de las comunidades afrodescendientes, los de las comunidades indígenas han sido forzados a desplazamientos masivos, que los han obligado a refugiarse en los mal llamados "cinturones de miseria", en donde comparten su pobreza y despojo, asumiendo otra territorialidad que no los liga con sus ancestros y los obliga a convertirse en ciudadanos de estrato uno, así como a disfrutar de los derechos y deberes que el incumplido y corrupto Estado colombiano tiene para todos aquellos que no formamos parte de la elite que acapara, controla y monopoliza el $90 \%$ de la riqueza que existe en el país.

\section{Justificación}

En nuestro país aún prevalecen rezagos de los modelos educativos (incluida la educación física) asimilacionistas y monoculturalistas, heredados del sistema colonial e impuestos por la clase dominante y por quienes se encargaron de la educación (especialmente la Iglesia católica). En el siglo XVI las instituciones encargadas de la educación de las clases altas exigían como condición indispensable, presentar los comprobantes de limpieza de sangre, imponiendo las pautas culturales de la etnia dominante y desconociendo completamente las raíces multiculturales de la población colombiana. El asimilacionismo propuso y oficializó la uniformidad cultural en la que los grupos subyugados adoptaron la lengua, los valores, las normas y la identidad de la cultura dominante, abandonando el pensamiento andino y adoptando la hegemonía cultural occidental con su racionalidad mercantilista. 
El modelo asimilacionista en el sistema educativo colombiano surge de la necesidad, de los colonizadores y posteriormente de las elites políticas, de que las otras clases sociales actuaran y pensaran de acuerdo a un "orden social" impuesto: justicia, ley, orden, gobierno, relaciones interpersonales y modos de producción, utilizando para ello instituciones como la escuela; aunque este sistema educativo fuera incoherente con las metas de la educación.

Hasta comienzos del siglo XX se trata de establecer un sistema educativo orientado a la formación de las personas, como ciudadanos que acepten toda norma civil y por tanto sean conocedores de sus derechos. Pero esta intención se fue desfigurando con los cambios de la economía nacional insertada dentro de un marco internacional. Por tanto, la formación tuvo que delimitarse hacia la producción de mano de obra eficaz, para circular como fuerza de trabajo, luego de terminar el ciclo básico educativo. Esta es la causa para que, desde mediados del siglo $\mathrm{XX}$, se asumiera un modelo educativo fundamentado en la tecnología, centrada en la "transmisión de conocimientos". El papel del Estado y de este modelo no tiene en cuenta la complejidad étnica ni cultural de quienes conforman la nación, sino que se repliega a las exigencias del sistema capitalista reforzando así un modelo social de homogenización.

Hasta hace muy pocos años se dio un cambio filosófico frente al modelo educativo impuesto por las necesidades de desarrollo económico. Surgieron entonces intelectuales, "seudointelectuales" e intelectualoides de escritorio que se preocuparon por buscar un modelo educativo que atendiera la formación de la persona en procura de lograr cierta libertad individual. Este cambio formal fue promovido por los seguidores de la escuela activa y del constructivismo, hecho que repercutió, también, en la forma de evaluación para lograr un acercamiento a las necesidades de los estudiantes reflejada en el imaginario economicista del docente o de cajero como la denomina Estanislao Zuleta.

Sin embargo, la actual crisis económica deja al descubierto las falsas promesas reformistas por esencia, del sistema educativo hegemónico y su modelo pedagógico que no dejó de ser asimilacionista y excluyente. Buscando ajustarse a las políticas trazadas por el neoliberalismo, promueve la profesionalización, el individualismo, el consumo, para un mercado ávido de alienados por el paraíso del capital, olvidando y dejando a un lado el principio fundamental de la educación: la formación de personas autónomas, independientes, libres. Así mismo, a pesar de haber transcurrido diez años de la Carta constitucional, queda en entredicho la autonomía de las comunidades, ya sean étnicas o no, puesto que sus organizaciones comunitarias, educativas y sociales son arrasadas bajo el emblema de la seguridad democrática, dando al traste con la intención que tuvieron los constituyentes: dejar un espacio escrito para la diversidad y la convivencia en la diferencia.

Desde la etnoeducación y la educación propia, es menester reconstruir la autonomía que hace factible la organización participativa y consciente de cada individuo en un grupo social; como uno de los elementos dinamizadores, al generar una actitud critica reflexiva y positiva, ausente en los hechos educativos que componen el modelo actual de la "Revolución Educativa", centrada en los famosos estándares de calidad, que dan al traste con derechos adquiridos a través de la Constitución y con el reconocimiento a la diversidad étnica y pluricultural de la población colombiana.

Desde estos referentes cabe anotar entonces, que el papel de la educación en general y de la etnoeducación física en particular, es el de posibilitar las condiciones para generar en las comunidades y colectividades, la participación democrática bajo una ética de equidad económica y ciudadana, que posibilite cambios radicales en los patrones culturales consumistas existentes, que persisten en invisibilizar y desaparecer a las comunidades étnicas; a quienes conciben la vida, y la vida humana, diferente al del proyecto racional de occidente y que manifiestan sus expresiones culturales desde la espiritualidad, y no desde la biofisiología del rendimiento o la higiene. 
Los hechos y la escasa producción teórica, muestran que la educación física en Colombia no ha salido del estado de postración que la mantiene el tránsito entre el modelo educativo hegemónico y excluyente de la diversidad cultural y étnica (más preocupado por formar consumidores para la apertura económica, que de las necesidades reales de miles de colombianos en la búsqueda de una equidad económica y el establecimiento de posibilidades de existencia entre las diversas comunidades étnicas y el resto del país), y el modelo del rendimiento deportivo, que la desplazó de las prácticas escolares, y le vende a millones de colombianos la falsa ilusión de salir de la pobreza algún día.

En contraposición a estos modelos hegemónicos, excluyentes y de competencia, surge el multiculturalismo como una opción de resistir y exigir la protección de la existencia como pueblos, comunidades, etnias, el derecho a la vida, a la diversidad cultural, con un principio básico: "el principio inspirador de la educación multicultural es sensibilizar a los individuos hacia la inherente pluralidad del mundo, la pluralidad de sistemas, creencias, estilos de vida, culturas, maneras de enfocar los acontecimientos históricos, etc. Complementan esta opción otros principios básicos como:

a) Respeto y protección a todas las culturas.

b) Derecho a la diferencia y a la organización de la sociedad de tal forma que exista igualdad de oportunidades y de trato.

c) Posibilidades reales de participación en la vida pública y social para todas las personas y grupos, con independencia de su identidad cultural, étnica, religiosa o lingüística.

El decreto 1122 de 1998 del Ministerio de Educación, reglamenta que el Proyecto Educativo Institucional (PEI), debe ser para la educación intercultural porque se imparte educación a una población pluricultural y multiétnica. Por lo tanto, la educación intercultural se requiere en toda la sociedad colombiana (no es exclusiva de los afrodescendiente e indígenas), lo que exige la presencia y reconocimiento de la otredad en los diálo- gos culturales, de saberes y prácticas sociales que han sido y siguen siendo excluidas o invisivilizadas en la vida del país.

Ahora bien, la Ley General de Educación define al currículo como el "conjunto de criterios, planes de estudio, programas, metodologías y procesos que contribuyen a la formación integral y a la construcción de la identidad cultural nacional regional y local", es decir que el verdadero cambio debe centrarse en aspectos actitudinales, cognitivos, de valores y creencias, que eviten las prácticas pedagógicas discriminatorias.

El planteamiento pedagógico propuesto comienza por reconocer la magnitud y la complejidad de la diversidad cultural, prácticas sociales y corporales; por lo tanto, de los múltiples horizontes de saberes de los unos y los otros, ya sean habitantes urbanos o rurales, que necesitan ser estudiados desde otros enfoques y concepciones, que fracturen o desquebrajen las rancias disposiciones administrativas y académicas (inscritas igualmente en el modelo hegemónico, excluyente y rendimiento de la inversión).

La reconceptualización de las tendencias pedagógicas, su aletargada o mecánica aplicación, replantean también, el asumir la diversidad como eje fundamental para comprender las características de las comunidades; colectividades híbridas y singulares que vienen elaborando otras propuestas de vida y educación por fuera del sistema educativo formal, y a las que la academia ignora o invisibiliza a nombre de la razón.

\section{La etnoducación física: un hecho social, un derecho social}

En párrafos anteriores se ha expuesto la afirmación de una educación hegemónica, asimilacionista, excluyente; como política clara y determinante del Estado colombiano, quien no ha convocado ni ha permitido, por ningún motivo, la participación de las fuerzas sociales que constituyen el país, en un proyecto de nación, de cultura, de educación, ca- 
yendo en un despotismo y autoritarismo que ha traído como consecuencia, la negación permanente a construirnos socialmente, a abortar y eliminar los procesos sociales que llevan implícitamente la constitución de subjetividad e intersubjetividad que permitan reformas reales con una población comprometida y defensora de los cambios realizados.

Los sectores dirigentes que constituyen la elite, han preferido la reforma a través de la ley; práctica heredada de la corona española y del general Santander que les permite arrancar las banderas de lucha, las ideas y propuestas de cambio a los sectores sociales, para llevarlas al Congreso, deformarlas, tergiversarlas y convertirlas en leyes, decretos, ordenanzas, acuerdos que no están dispuestos a cumplir por nada del mundo, pero rompiendo eso sí, los procesos sociales, que generan en el imaginario de los gobernados: que son las leyes y la práctica social, las que nos van a cambiar de concepción.

Por eso encontramos una gran cantidad de legislación sobre los hechos sociales, culturales, económicos, jurídicos, que hacen parte de la vida de los colombianos. Parece ser que hubiera más leyes que hechos por estudiar. A esta enfermedad legislativa, no escapa el hecho social conocido como educación física, como tampoco escapa al modelo educativo hegemónico, asimilacionista y excluyente del cual hace parte.

En 1991, en el juego de la "democracia representativa" con la expedición de la Constitución Política de Colombia, quedó condensada la Educación Física como un derecho social de todos los ciudadanos colombianos. Por ley pasó de ser una simple asignatura al privilegio de derechos como la salud, la vivienda, el trabajo, etc, a los cuales el Estado debe asignarle recursos, vigilar y garantizar su acceso. Pero como es el resultado de la enfermedad legislativa y no de un movimiento social que lo reivindique, catorce años después sale una disposición: que elimina los profesores de educación física de la educación básica primaria, expedida por el alcalde, Dr. Antanas Mockus, $y$ aplicada al pie de la letra por la Secretaría de Educación Distrital, y no pasa nada, el magisterio distrital no opone ninguna resistencia, las facultades de educación física no se dan por enteradas, $y$ se acepta tal arbitrariedad, violatoria de un derecho social.

Asimismo, quedó registrado en el papel firmado por los constituyentes y posteriormente por los congresistas de turno: que somos un país pluriétnico y multicultural en donde no debe existir un solo modelo educativo. Posteriormente aparecen las disposiciones que reglamentan eso hecho jurídico trascendental para el juego de la democracia representativa. En la actualidad, con el famoso referendo, se pretende desmotar el reconocimiento y borrar de la vida del país nuevamente las diferencias étnicas y culturales que tanto desorden le causa a la tranquilidad de las élites. Sin embargo a diferencia de lo sucedido con la educación física (en donde educadores de lo físico y educados físicamente en la alienación causada por el modelo educativo de que son víctimas, que no tienen conciencia de lo adquirido

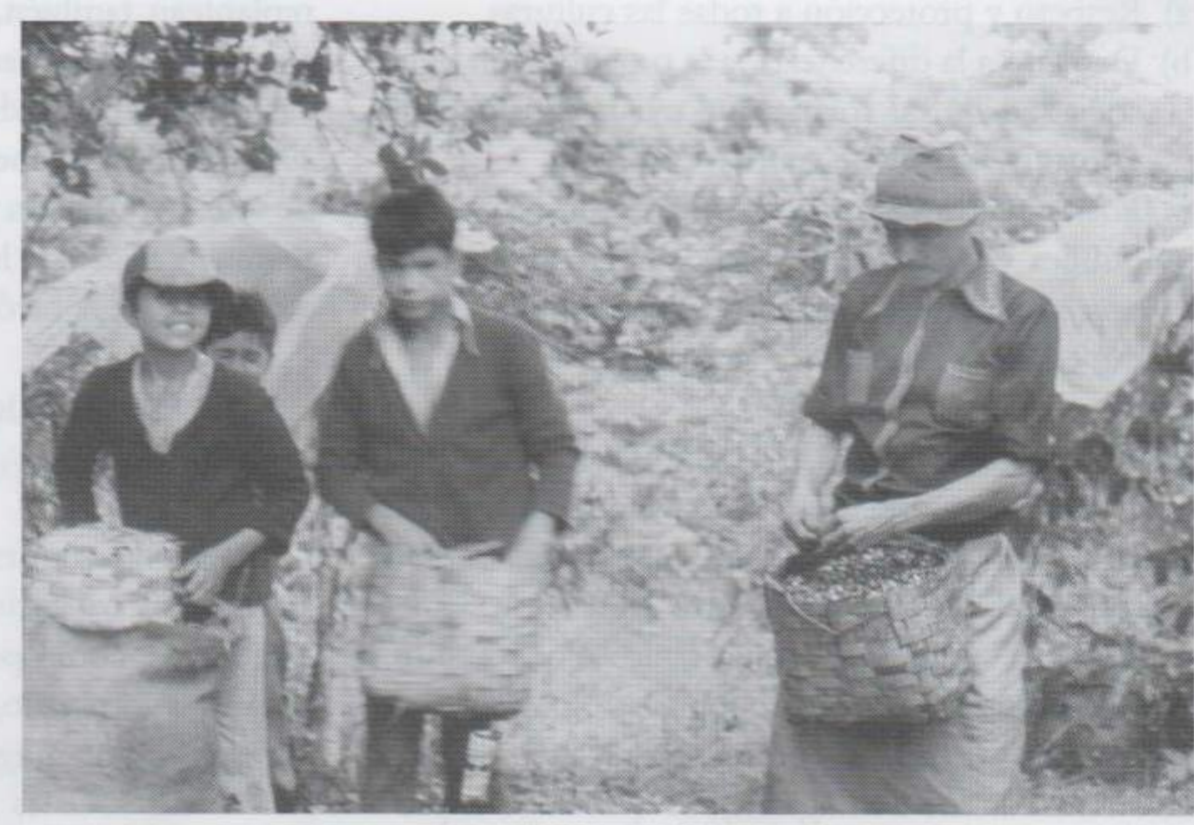

Por: Mario G. Loaiza Padilla 
por ley), la población indígena, afrocolombiana, gitana, raisal, muestra su gran descontento y exige el cumplimiento del compromiso registrado en la Constitución, a través de la existencia como pueblo, comunidad o etnia, organizada en un movimiento social.

Ahora bien, como se ha expuesto que no son las leyes las que cambian o modifican las conciencias y menos las prácticas sociales de los legislados, sino los procesos sociales que adelantan las comunidades a través de los diferentes movimientos y luchas, es pertinente aclarar que la propuesta de una educación multicultural, en la que se incluye la etnoeducación física, no se fundamenta en la legislación que está sujeta a la endemia expuesta, sino que utiliza los espacios abiertos por lo jurídico. Es decir, su fundamento lo constituye los principios enunciados en páginas anteriores, en el derecho propio a tener una educación propia, y no en la voluntad o bondad del legislador de turno.

Eso exige replantear la concepción de la educación física occidentalizada, retomando el pensamiento ancestral del mundo andino o africano para la elaboración de otras explicaciones mediante la generación de hechos sociales que tengan significantes y significados en asideros reales, de confrontación de intereses, de diálogos culturales, de saberes presentes en las prácticas culturales y sociales de las comunidades.
No podemos seguir acuñando las prácticas y conceptos de una educación física reduccionista que muestra los gestos y expresiones del movimiento humano como resultado de gastos energéticos, procesos biofisiológicos, anaeróbicos, etc., despojándolos de su contenidos culturales, sociales, filosófi$\cos$, de las relaciones que establece el ser humano con las otras formas de vida y que le permiten vivir. Es decir, no debemos agenciar un autismo cósmico total, en donde el desarrollo humano no esté regulado por las contradicciones de las complejas fuerzas del universo, y en la que la actividad física sea solo muestra de sudor, olor, dolor, domesticación corporal, consumo de modas y productos para estar in, "saludable" o rehidratarse.

El despojo que hacen estas prácticas tiene como finalidad, precisamente subir al mismo bus a todos los colombianos, indistintamente de sus cosmogonías, etnias, prácticas corporales, religiosas, culturales y estado jurídico, para recetarles la formula mágica que les producirá los mismos efectos: la alineación y la felicidad que da el sometimiento.

Sectores amplios de la población colombiana compraron hace rato el tiquete de viaje, otros añoran subirse al bus. Se endeudan o hipotecan sus conciencias, creencias y hasta el derecho de ser humanos.

¿Por qué seguir pensando y practicando una educación física que desconoce y excluye en su concepción y práctica social, los hechos reales y las realidades de la población colombiana?

\section{Bibliografía}

MINISTERIO DE EDUCACIÓN NACIONAL. Decreto 1122 de 1998. Bogotá. p. 2.

PÁRAMO, Pablo. (2000). Colombia necesidad de utopia. En: Magazín Dominical el Espectador (marzo). Bogotá.
ZULETA, Estanislao. (1996). Educción y democracia. Un campo de combate. Bogotá. 